\title{
Unusual case of Boerhaave syndrome, diagnosed late and successfully treated by Abbott's T-tube
}

\author{
Mario Santini, MD, ${ }^{a}$ Alfonso Fiorello, MD, ${ }^{a}$ Salvatore Cappabianca, MD, and \\ Giovanni Vicidomini, MD, PhD, ${ }^{a}$ Naples, Italy
}

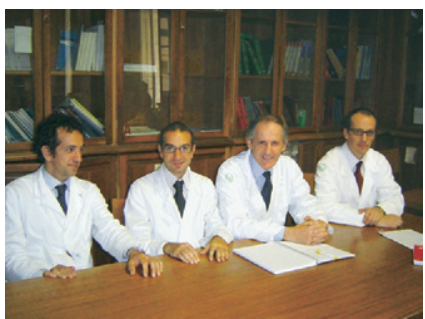

Drs Vicidomini, Fiorello, Santini, and Cappabianca (left to right) pontaneous rupture of the esophagus is a rare and potentially fatal condition with an overall mortality rate of $20 \%$ to $50 \%$. We report a rare case of Boerhaave syndrome that was successfully treated with Abbott's T-tube.

\section{Clinical Summary}

A 33-year-old man presented to the emergency department of a local hospital with vomiting followed by hematemesis, chest pain, dyspnea, and fever. The chest radiography and computed tomographic (CT) scan revealed opacification of the right side of the chest. Two chest tubes were inserted with drainage of purulent fluid and regression of the clinical symptoms. A CT scan performed 2 days later revealed contrast-medium extravasation from the esophagus into the right hemithorax (Figure 1, A). The patient's condition deteriorated 24 hours later; he showed fever and a leucocytosis count of $13,000 / \mu \mathrm{L}$. He was transferred to our institution 7 days after perforation, and a right thoracotomy was performed on the same day. At operation, a fibrin peel imprisoned the lung. After decortication, a complete reexpansion of the lung was obtained. However, a $3.5-\mathrm{cm}$ transmural perforation of the esophagus was found, just below the azygos vein. The defect was débrided, and a T-tube was positioned. The chest was closed with multiple chest tubes in place. One drainage tube was placed in proximity to the T-tube, and the other tubes were placed in the pleural cavity and mediastinum (Figure 1, $B$ ). The patient was fed parenterally through a central venous line, and systemic antibiotics were administered. The postoperative course was unremarkable except for sporadic peaks of fever (up to $40^{\circ} \mathrm{C}$ ) that stopped after suspension of antibiotics. The pleural and mediastinal drains were gradually removed. The T-tube (Figure 2, A) was removed in 2 steps 3 weeks later. First, we cut and removed the portion of the short branch of the T-tube through an esophagoscopy; the large branch was removed 2 days later. After repeated negative esophagrams (Figure 2,B), the patient resumed a regular diet and was discharged on postoperative day 38 without symptoms.

From the Thoracic Surgery Unit ${ }^{\mathrm{a}}$ and Department of Radiology, ${ }^{\mathrm{b}}$ Second University of Naples, Italy.

Received for publication April 17, 2007; accepted for publication April 26, 2007.

Reprint requests: Mario Santini, MD, Chirurgia Toracica-Seconda Università di Napoli Piazza Miraglia, 2, I-80138 Naples, Italy (E-mail: mario. santini@unina2.it).

J Thorac Cardiovasc Surg 2007;134:539-40

$0022-5223 / \$ 32.00$

Copyright (C) 2007 by The American Association for Thoracic Surgery doi:10.1016/j.jtcvs.2007.04.033

\section{Discussion}

Boerhaave syndrome is the most lethal perforation of the gastrointestinal tract. ${ }^{1}$ The combination of gastric acid and digestive enzymes causes mediastinitis and sepsis, which are fatal in most untreated cases. Although approximately 300 cases of Boerhaave syndrome are reported in the literature, our case is interesting for 4 points summarized in the acronym "S.T.O.P." (ie, "stop! pay attention"): Site, Time, Operation, Prognosis. Site: In $80 \%$ of cases, the rupture takes place in the lower left portion of the esophagus, 2 to $3 \mathrm{~cm}$ proximal to the gastroesophageal junction. In this case, the tear was localized in the middle right portion of the esophagus just below the azygos vein. Time: Diagnosis can be difficult, and prompt recognition is vital to ensure appropriate treatment. Although our patient presented with specific symptoms of Boerhaave syndrome, he was first treated for empyema. The CT scan performed 7 days after perforation revealed contrast extravasation from the esophagus into the right hemithorax. The patient

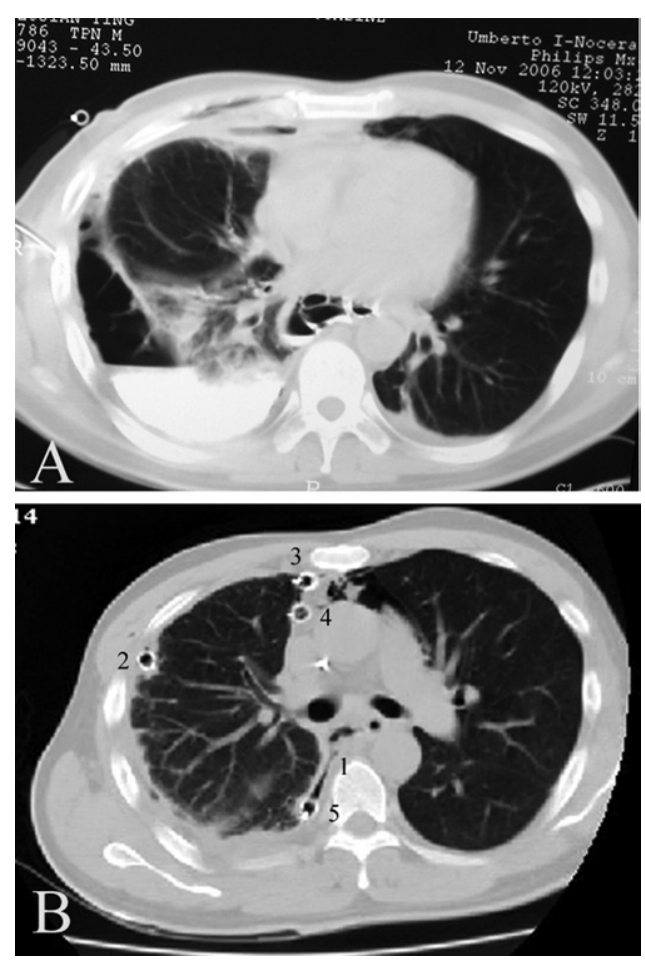

Figure 1. A, CT scan shows contrast extravasation from the esophagus into the right hemithorax with lung compression. B, T-tube is positioned (1), and the chest is closed with multiple tubes in place: in pleural cavity $(2,3)$, mediastinum (4), and next to the T-tube (5). 

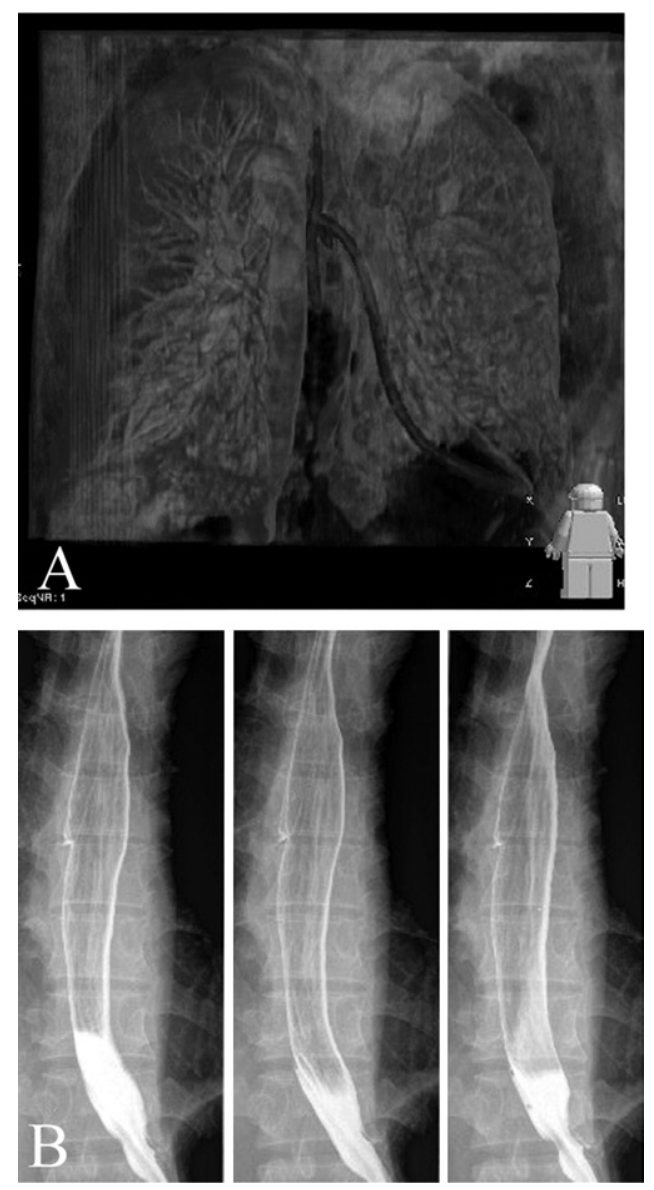

Figure 2. A, Three-dimensional reconstruction of CT scan reveals T-tube localized in the middle right portion of the esophagus just below the azygos vein. The short limb of the T-tube traverses the esophagus, and the large limb exits from the chest. B, Esophagram demonstrates the healing of the tear.

was transferred to our institution to undergo a thoracotomy. In 2 of 21 patients seen by Lawrence and colleagues, ${ }^{2}$ the diagnosis of Boerhaave syndrome was made more than 2 weeks after the rupture. However, in these cases it was sufficient that the débride- ment and drainage of empyema were performed by thoracoscopy. Operation: Management is controversial for the heterogeneity of causes. ${ }^{3}$ Surgical repair of the rupture is appropriate in early cases $(<24$ hours after perforation), and nonoperative management is appropriate in patients with perforations who are seen later. ${ }^{4}$ In this case, we excluded the tear repair because the mucosa appeared too friable to hold sutures. Furthermore, the presence of mediastinal sepsis may lead to a high risk of anastomosis leaking. Thus, two strategies were possible: esophageal exclusion or T-tube drainage, as described by Abbott and colleagues. ${ }^{5}$ We did not choose esophageal exclusion because it requires an additional cervical approach and is associated with a consistent morbidity, including inhalation pneumonia, stenosis, or asynchronism in repermeation. A large soft T-tube was inserted through the perforation. The short branch traversed the esophageal lumen, and the large branch exited from the chest. This allowed control of the fistula and drainage of esophageal secretions and refluxed gastric materials. Prognosis: Patients who undergo surgical repair within 24 hours from injury have a $70 \%$ to $75 \%$ chance of survival. This decreases to $35 \%$ to $50 \%$ if surgery is delayed more than 24 hours. Despite the free interval between the acute episode and the operation, our patient survived and had no other symptoms.

\section{Conclusions}

According to our experience, T-tube drainage should be considered as an alternative to other surgical strategies used in Boerhaave syndrome.

\section{References}

1. D'Journo XB, Doddoli C, Avaro JP, Lienne P, Giovannini MA, Giudicelli $\mathrm{R}$, et al. Long-term observation and functional state of the esophagus after primary repair of spontaneous esophageal rupture. Ann Thorac Surg. 2006;81:1858-62.

2. Lawrence DR, Ohiri SK, Moxon RE, Tonsed ER, Fountain SW. Primary esophageal repair for Boerhaave syndrome. Ann Thorac Surg. 1999;67:818-20

3. Jones WG, Ginsberg RJ. Esophageal perforation: a continuing challenge. Ann Thorac Surg. 1992;53:534-43.

4. Flynn E, Verrier ED, Way LW, Thomas AN, Pellegrini CA. Esophageal perforation. Arch Surg. 1989;124:1211-5.

5. Abbott OA, Mansour KA, Logan XD, Hatcher CR, Symbas PN. Atraumatic so called spontaneous rupture of the oesophagus. A review of 47 personal cases with comments on a new method of surgical repair J Thorac Cardiovasc Surg. 1970;59:67-83. 